\title{
Ultra Wideband Multiple-Input Multiple-Output Radar
}

\author{
Hammad A. Khan, Wasim Q. Malik, David J. Edwards, Christopher J. Stevens \\ Department of Engineering Science, University of Oxford \\ Parks Road, Oxford OX1 3PJ, UK. \\ Email: \{hammad.khan, wasim.malik, david.edwards, christopher.stevens\}@eng.ox.ac.uk
}

Key Words: Ultra Wideband (UWB), MIMO, radar.

\section{ABSTRACT}

The utilization of ultra wideband (UWB) signals enables the radar designer to solve the most important problems of radar target observation. The extremely wide bandwidth enables greater information to be obtained due to high time resolution and the frequency dependence of the scattering centers over this large bandwidth. Increase in the radar's signal bandwidth can improve radar performance by providing better range measurement accuracy, improving the target identification and tracking capability, improving radar immunity to passive interference, and enhancing radar countermeasure against narrowband electromagnetic signal interference. Recently there have been many advances in multiple-input multiple-output (MIMO) antenna systems in communications. These diversity systems have been shown to have the potential to dramatically improve the performance of the communications systems. Unlike the traditional beamforming approach, which uses highly correlated signals of an array of transmitting or receiving antenna elements to collimate a beam towards a certain direction in space, MIMO capitalizes on the independence between signals from different transmitters and on the diversity of target scattering to improve the information received from the response. Motivated by the advances and benefits of MIMO in communications and advantages of using UWB signals, this paper presents the experimental investigation of UWB-MIMO radars. The analysis of such radars has been carried out to demonstrate its promising features in terms of better target identification and improved signal to noise ratio (SNR).

\section{INTRODUCTION}

There are four metrics which determine the performance of a radar: reliability of detection, accuracy of target parameter estimation, resolution of multiple targets and ambiguity of target estimates [1]. Signal to noise ratio (SNR) affects the reliability of detection and accuracy of estimation. The signal bandwidth affects all performance measures. Signal waveform affects estimation ambiguity and repetition of waveforms affects detection, estimation and ambiguity [2]. The utilization of ultra wideband (UWB) signals improves the target resolution and accuracy of parameter estimation. UWB signals are less prone to passive interference due to their large bandwidth and have low probability of intercept and innocuous electromagnetic operation due to their low transmitting power. UWB radar has been a subject of research for a long time [3, 4]. But many areas are still being researched due to the benefits of UWB radars [5]. This paper explores the use of UWB signals together with a spatially distributed multiple transceiver scheme in radars. Multiple transceivers (or multiple signaling) stem from the use of spatial diversity in communication systems. In a communication system, four diversity schemes have been in use: time diversity, frequency diversity, polarization diversity and space diversity. Similar schemes can be used for radars. Time is a limiting commodity in a target tracking radar and hence not an efficient technique. The use of polarization has been shown to have its benefits in UWB radars [6], but its capabilities are limited by the scattering properties of the target. Use of multiple frequency bands can be used in narrowband radars but it requires the use of further bandwidth, which would otherwise be used for improving the range resolution. The use of spatially distributed multiple transceivers is limited only in terms of the usage of more space for implementation, but it improves the information extraction from the target by viewing the target from multiple angels. Coupling the multiple transceiver scheme with UWB signals yields fine range resolution along with the benefits of multi-aspect imaging.

Radars with more than one transmitting or receiving antenna elements have been built and tested widely [7]. An array of radar elements is used with highly correlated signals to form a controllable beam, which can be collimated in a certain direction to scan the desired space. Such radars are known as phased array radars and the process is called beamforming [8]. Beamforming uses highly correlated signals at the radar antenna elements. Instead of beamforming, which views one aspect of the target at a time, some work is being done in multiple-input multiple-output (MIMO) radar [9-12]. MIMO radar uses spatially distributed multiple transceivers. It capitalizes on the independence of transmitted and received signals and on the diversity of target scattering by viewing the target from multiple aspects. It is known that only very small changes in viewing angle causes large fluctuations in radar cross section (RCS) because the reflected energy depends heavily upon the range as well as the receive look-angle of the target [2]. This implies that while maximizing the received energy in radars, imaging the target with different orientations and appropriately combining the received signals could lead to 


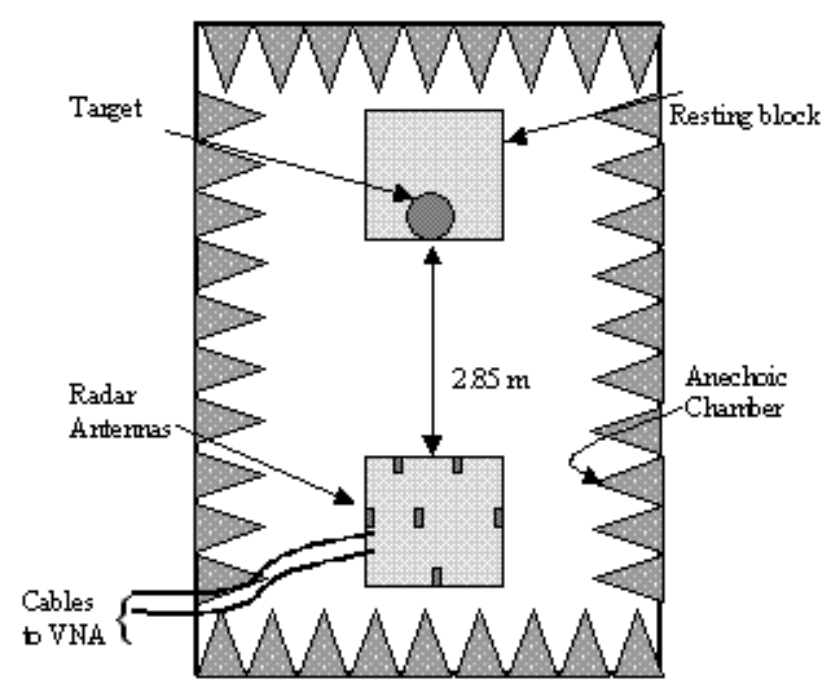

Fig. 1. Radar and target in the anechoic chamber (schematic plan view).

better information from the target. MIMO radar is inspired mainly by the synthetic impulse and aperture radar (SIAR) [13]. In SIAR, the sparse array antenna elements emit orthogonal waveforms, utilizing a time-space beamforming; improving the angular resolution by the use of sparse arrays. SIAR achieves the advantages of wideband radar using narrowband signals at individual antenna elements. But it suffers from the same performance degradation that the phased array radar is prone to: fading due to target's orientation fluctuation. Basic theoretical models of MIMO radar have shown its advantages over traditional beamforming approach $[9,10]$.

This paper presents the initial experimental results and analysis of UWB-MIMO radar. A set of measurements of UWB-MIMO radar signals was conducted using a vector network analyzer (VNA). A metallic target (a cylinder) was imaged with the radar in an anechoic chamber. The target response was analyzed to compare MIMO radar with its variants. Results show that the MIMO radar outperforms its counterparts and provides better target identification showing the promise in this new architecture of radars. The results also show that much more insight is needed to find out why some channels of the MIMO radar system perform better than the others. The aim of the experiments was to analyze initial measurements for UWB-MIMO radar in a non-multipath and less dense environment using simple signal processing tools and to establish the advantages achieved with UWB-MIMO radar. The initial experimental work has set the stage for investigation into an unexplored and promising new radar architecture.

\section{EXPERIMENTAL SETUP}

Fig. 1 shows the plan view of the experimental set-up. Both the target and the radar antennas were housed in an anechoic chamber and the antenna cables were connected to a VNA outside the chamber. Antennas and the target were supported by $4 \mathrm{~m}$ high resting blocks. The resting blocks were separated by $2.85 \mathrm{~m}$. This distance was the range of the radar.

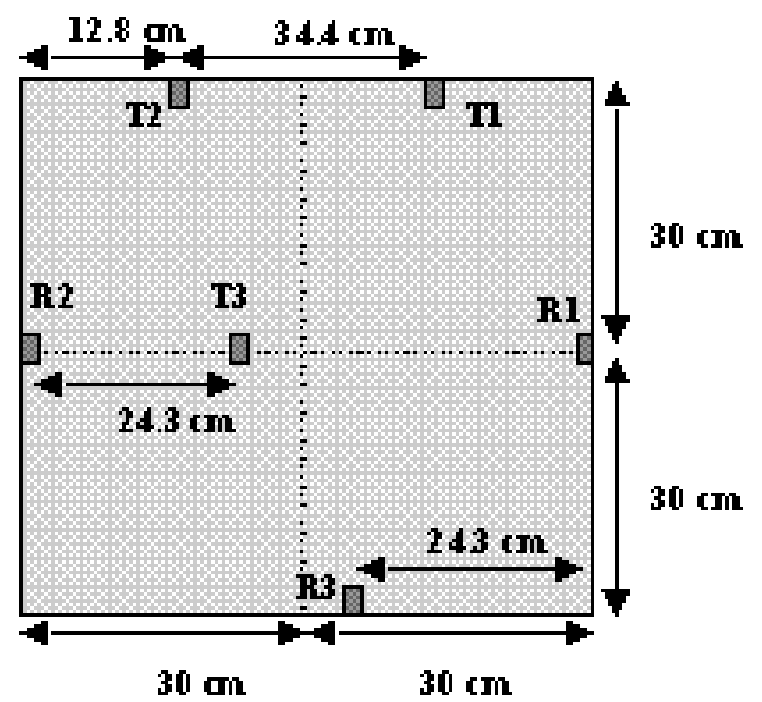

Fig. 2. MIMO antenna configuration. $\mathrm{T}$ and $\mathrm{R}$ denote different transmitters and receivers respectively.

Three transmit and three receive antenna positions on the antenna block were positioned as shown in Fig. 2. A minimum separation of antennas was maintained in order to avoid cross coupling and ensure the decorrelation of the channels. For analysis, same simulated UWB signal was sent over all the channels to simplify the signal processing. The target was a metallic cylinder of height $30 \mathrm{~cm}$ and diameter 9 $\mathrm{cm}$. Three sets of measurements were performed in the anechoic chamber. The first experiment sought to image the target alone. The second was the cylinder in the presence of clutter and the third measurement was without any target to correct for any unwanted reflections. Only one set of transmitter and receiver was connected to the VNA at a time and the positions of the antennas were changed to measure data for all permutations of transmitters and receivers. The clutter-based measurements are not included in this paper and will be a part of another publication with details of the clutter rejection algorithms used.

In performing VNA based measurements, the VNA acts both as a transmitter and a receiver. The antennas used in the measurements at the transmitter and the receiver ends are Vivaldi antennas [14].

The channel was sounded over a frequency range of 1.99 $\mathrm{GHz}$ to $10.6 \mathrm{GHz}$ in accordance with the FCC rules for UWB communications and systems [15] and the amplitude of transmission (S21 parameter of the VNA) was measured. The signals were combined assuming a stationary channel.

\section{MIMO PROCESSING}

The above experiment gave the transfer functions of three systems: anechoic chamber without any target, anechoic chamber with the target and anechoic chamber with target in the presence of clutter. As noted above, the clutter-based measurements are not included in this paper. Since the data was measured on one transmit-receive system at a time, using highly directional antennas, the signals must be combined in an appropriate manner for the MIMO system in order to get 


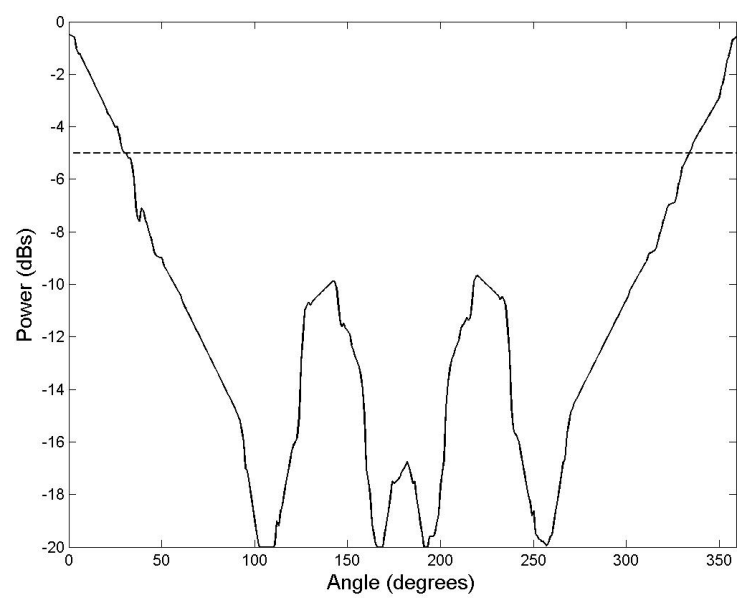

Fig. 3. Normalized Vivaldi antenna azimuth pattern.

full benefits of the multiple transceivers. There are wellestablished methods of combining signals in communications systems [16].

\subsection{Antenna Radiation Pattern}

Fig. 3 shows the antenna azimuth pattern of the Vivaldi antennas used in this work. The $5 \mathrm{~dB}$ power of the antenna is within $30^{\circ}$ of both the azimuth. Elevation pattern is not relevant to the analysis as all the antenna elements are in the same plane. This information is important when combining the signals at the receiver. The angle of view of the antenna element can be of great significance due to variation in the power received at various look-angles.

\subsection{Combining Techniques}

The most popular diversity combining schemes are Selection Combining (SC), Equal Gain Combining (EGC) and Maximal Ratio Combining (MRC) [16]. In EGC, all the received signals are co-phased before being added. The extra phase accrued by each transmit-receive path is calculated based upon the excess path length traversed by the signal measured with respect to the distance between the two resting blocks shown in Fig. 1. In MRC, the weights of the signal are proportional to the SNR at the receiver before they are cophased. While MRC only offers an advantage of about $1 \mathrm{~dB}$ over EGC in a communication channel [16], it might have an effect of considerably improving the range accuracy due to the variation of antenna power with the receive look-angle (Fig. 3 ). Before carrying out the analysis, target frequency response from any unwanted reflections off the anechoic chamber walls was corrected for. Channel transfer functions for different channels in the MIMO radar were measured using the VNA. Simulated UWB pulses were then used in Matlab ${ }^{\circledR}$ to find the target response. Fig. 4 shows the simulated pulse of $0.10 \mathrm{~ns}$ duration used to generate the required frequency range of 1.99 to $10.6 \mathrm{GHz}$. This pulse was obtained by using a Gaussian function. For additional pulse shaping, unwanted frequencies in the transfer function of the pulse were suppressed and the final time domain pulse obtained by inverse Fourier transform.
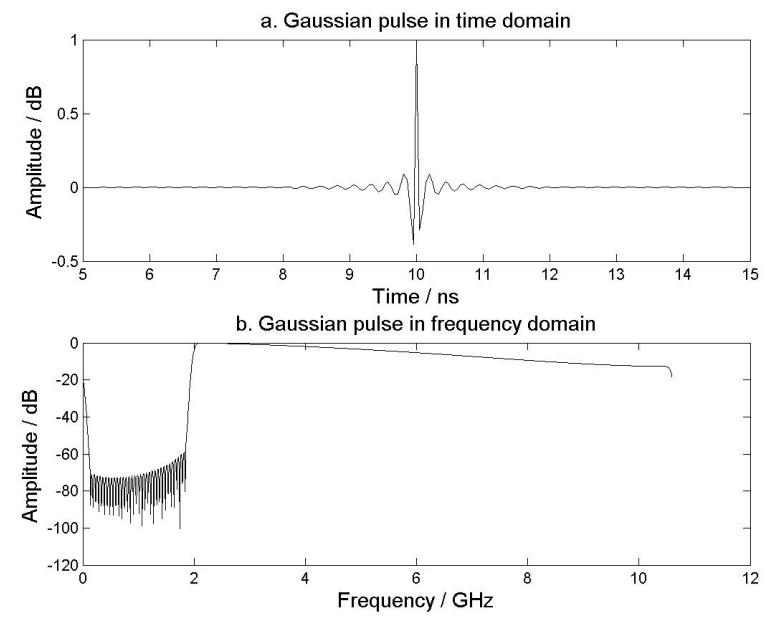

Fig. 4. Ultra-short pulse with the required frequency range of $1.99-10.6 \mathrm{GHz}$. The basic pulse shape is derived from a Gaussian function.

The sidelobes of the resulting pulse were windowed using a Gaussian window [8] to obtain the shape shown in Fig. 4. The $-10 \mathrm{~dB}$ frequency range for this pulse is 1.99 to $10.6 \mathrm{GHz}$ in agreement with the regulation requirements of UWB [15].

Let $H(f)$ be the (corrected) frequency response of the target obtained by the VNA and $X(f)$ be the transfer function of the pulse as shown in Fig. 4. Then the target response, $Y(f)$ to this pulse is given as

$$
Y(f)=H(f) \cdot X(f)
$$

And the time response is given as

$$
y(t)=\sum_{\text {all } f} Y(f) e^{-j 2 \pi f t}
$$

The target's response to this pulse is given in Fig. 5 using EGC and MRC. The response using ECG gives an erroneous value of $3.05 \mathrm{~m}(6.10 / 2$ from the graph) for the range. The error in this result is around $7 \%$. This clearly does not achieve the expected range resolution of UWB radars. The high error can be due to the same SNR addition used in EGC. The Vivaldi antenna azimuth pattern (Fig. 3) reveals that different receive look-angles have different $\mathrm{SNR}$ for the received signals. SNR has thus to be weighed before combining the signals at the receivers. This type of combining is MRC gives a better range estimate of $2.90 \mathrm{~m}$ ( $1.8 \%$ error). The target response in both the figures reveals two distinct and close peaks, which can be used to find out information about the target, e.g. the its cross sectional size.

The horizontal axis in Fig. 5 represents the distance, $d$, corresponding to the time, $t$, where the receiver scans for signals all the time and $d=c t$; $c$ being the speed of light. A strong signal at time $t$ comes from the transmitted signal that has been reflected from an object at a distance $d / 2$ from the radar. The vertical axis in the figure represents the amplitude of the received signals in $\mathrm{dBs}$. The threshold of the returned signals is set to be $20 \mathrm{~dB}$ below the maximum. This is shown as black dotted line in the figures. The rest of the response is considered as noise. There is no signal above the threshold until after $5.95 \mathrm{~m}$ for ECG and $5.7 \mathrm{~m}$ for MRC. 


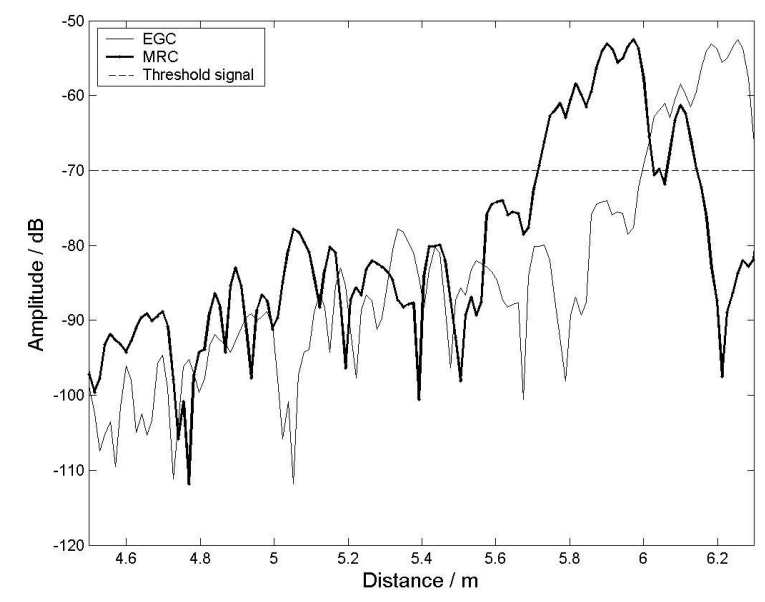

Fig. 5. Target response using equal gain combining (ECG) and maximal ratio combining (MRC). Threshold signal is shown by a dotted line at $-70 \mathrm{~dB}$.

\subsection{Correlation Coefficients}

The MIMO radar sets up communication channels in the anechoic chamber described in this paper. Every transmitreceive antenna pair has a channel set up between them. This gives rise to nine (ideally) independent channels. In a multiple transceiver system, such as this one, it is vital that the received signals (and the communication channels) are independent of each other [9]. This independence is measured by computing the correlation coefficient between the signals received at different receivers or between different communication channels. The correlation coefficient between two signals (or channels) $x$ and $y$ can be calculated as

$$
\frac{E[x y]-E[x] E[y]}{\sqrt{\left(E\left[x^{2}\right]-E[x]^{2}\right)\left(E\left[y^{2}\right]-E[y]^{2}\right)}}
$$

where $E[$.$] is the expected value of a variable. Here x$ and $y$ are assumed to be random variables. A correlation value of 0.7 is used as the threshold below which the signals are considered independent [17]. Table I shows the correlation coefficients between all the nine channels set up in this experiment. It is observed that apart from one anomaly (between T1R3 and T3R1), the correlation values are well below the 0.7 threshold, hence showing the validity of the implemented multiple transceiver scheme.

\section{COMPARATIVE RESULTS}

UWB radars mostly use single transmit-receive systems or multi-static single transmit-multiple receive systems [3]. Some systems use multiple pulses at the transmitter and pulse integration at the receiver to enhance the SNR. This section compares different approaches with the proposed MIMO radar.

\subsection{MIMO vs. Single Transmit-Receive System}

Fig. 6 shows the comparison of MIMO radar with pulse integration by two single transmit-receive systems. Ideal
Table I

Correlation coefficients of the different channels that are set up in the MIMO radar. An $\mathbf{x}$ indicates that the correlation is between the same channel and hence not meaningful. (Note that the right upper triangle of the table is redundant).

\begin{tabular}{|c|c|c|c|c|c|c|c|c|}
\hline & $\underline{\underline{\Xi}}$ & $\stackrel{\widetilde{\Xi}}{\vec{\Xi}}$ & $\underline{\underline{G}}$ & $\underset{\widetilde{N}}{\bar{N}}$ & $\frac{\widetilde{\widetilde{V}}}{\mathbb{N}}$ & $\stackrel{\overparen{\pi}}{\stackrel{N}{N}}$ & $\overline{\underline{m}}$ & $\stackrel{\widetilde{\widetilde{m}}}{\tilde{m}}$ \\
\hline T1R1 & $\mathbf{x}$ & & & & & & & \\
\hline T1R2 & .19 & $\mathbf{x}$ & & & & & & \\
\hline T1R3 & .22 & .33 & $\mathbf{x}$ & & & & & \\
\hline T2R1 & .49 & .45 & .47 & $\mathbf{x}$ & & & & \\
\hline T2R2 & .56 & .20 & .51 & .56 & $\mathbf{x}$ & & & \\
\hline T2R3 & .51 & .19 & .56 & .52 & .56 & $\mathbf{x}$ & & \\
\hline T3R1 & .17 & .27 & .73 & .49 & .37 & .57 & $\mathbf{x}$ & \\
\hline T3R2 & .23 & .21 & .68 & .47 & .36 & .57 & .83 & $\mathbf{x}$ \\
\hline T3R3 & .48 & .24 & .55 & .44 & .44 & .58 & .54 & .54 \\
\hline
\end{tabular}

coherent integration was used which increases the SNR at the receiver by a factor equal to the number of pulses integrated [2]. The figure reveals the advantage of multi-aspect radar by observing the variation in range estimates and SNR with different transceivers. It should also be noted that pulse integration is not ideal in practice due to receiver inefficiencies and some loss of energy occurs in integrating the returned pulses due to their different times of arrival and coherent integration becomes difficult to realize [2].

\subsection{MIMO vs. SIMO and MISO}

In Fig. 7, the MIMO radar is compared with a single-input multiple-output (SIMO) radar and multiple-input single-output (MISO) radar. The figure shows that there is no significance difference in the information content between MISO and SIMO radars. Fig. 6 and Fig. 7 also reveal the promising advantages of MIMO radar for enhanced SNR, better range accuracy, and the possibility of obtaining more target information. The last improvement stems from the highly detailed signal peaks of MIMO. Given a better representation of the target responses (using time-frequency analysis), this information from the MIMO radar can be used to find out the target size and orientation if its shape is known or vice versa and fairly hidden targets can also be detected and identified. These issues will be addressed in a later publication.

\section{CONCLUSIONS}

This paper has presented experimental and simulation results of the preliminary work on UWB-MIMO radar systems with three transmit and three receive antennas. The MIMO radar has been compared with its variants - the pulse integration radar and the MISO and SIMO radars. The results show the promising features of UWB-MIMO radar, which will be explored further in terms of clutter rejection and target identification in dense environments before realizing the full potential of these types of radars. 


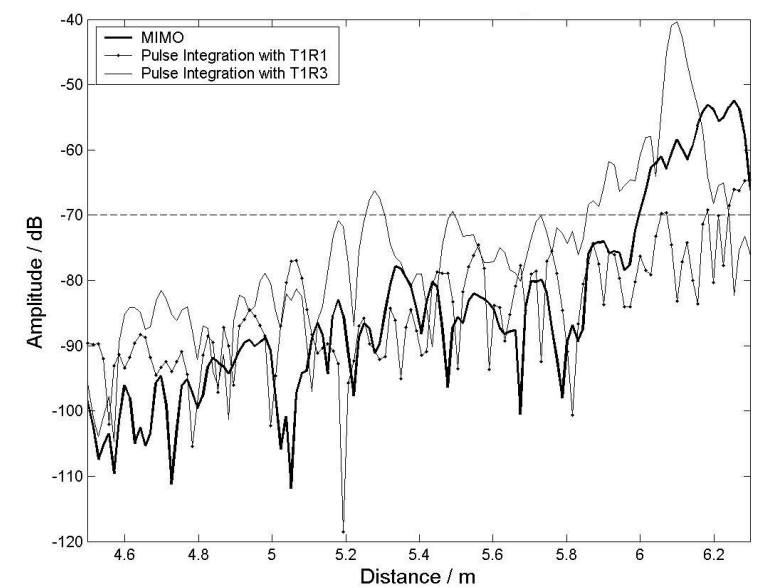

Fig. 6. Comparison of MIMO radar and pulse integration. Two different pulse integration transceivers are used. Threshold signal is shown by a dotted line at $-70 \mathrm{~dB}$.

\section{ACKNOWLEDGEMENTS}

Hammad Khan's work is supported by the Rhodes scholarship.

\section{REFERENCES}

[1] W. M. Siebert, "A radar detection philosophy," IEEE Trans. on Information Theory, vol. 2, pp. 204-221, 1956.

[2] M. I. Skolnik, Introduction to Radar, 3rd ed: McGrawHill International Editions, 2001.

[3] J. D. Taylor, Ultra-wideband Radar Technology: CRC Press, 2000.

[4] L. Y. Astanin and A. A. Kostylev, Ultra-wideband Radar Measurements-Analysis and Processing: IEE, 1997.

[5] I. J. Immoreev and J. D. Taylor, "Future of Radars," in 2002 IEEE Conference on Ultra Wideband Systems and Technologies, 2002, pp. 197-199.

[6] Y. Xu, "Random noise polarimetry: theory and applications towards subsurface probing." $\mathrm{PhD}$ Thesis: University of Nebraska-Lincoln, 1997.

[7] A. Farina, Antenna Based Signal Processing Techniques for Radar Systems. Norwood, MA: Artech House, 1992.

[8] S. Haykin, J. Litva, and T. J. Shepherd, Radar array processing, 1st ed. New York: Springer-Verlag, 1993.

[9] E. Fishler, A. Haimovich, R. Blum, L. Cimini, D. Chizhik, and R. Valenzuela, "MIMO radar: An idea whose time has come," in Proc. of the IEEE Int. Conf. on Radar. Philadelphia, PA, April 2004.

[10]E. Fishler, A. Haimovich, R. Blum, L. Cimini, D. Chizhik, and R. Valenzuela, "Spatial diversity in radarsmodels and detection performance.," IEEE Trans. on Signal Processing, 2004, In Press.

[11]D. Rabideau, "Ubiquitous MIMO digital array radar," in Proc. of the Asilomar Conference on Signals, Systems and Computers, November 2003.

[12]P. Bidigare, "MIMO Capacity of Radar as a Communication Channel," in Eleventh Annual Workshop

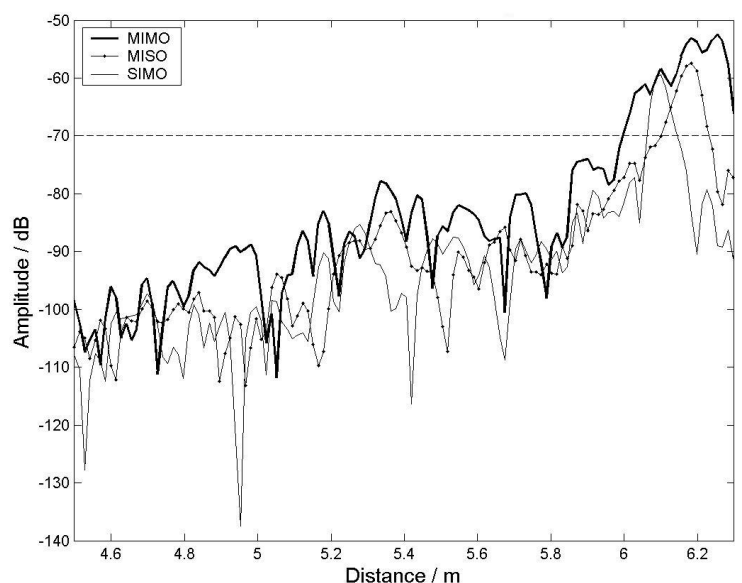

Fig. 7. Comparison of target responses from MIMO, MISO and SIMO radars. Threshold signal is shown by a dotted line at $-70 \mathrm{~dB}$.

on Adaptive Sensor Array Processing. Lexington, Massachusetts, 2003.

[13]C. Baixiao, L. Hongliang, W. Yajun, and W. Jun, "Analysis and experimental results on sparse array synthetic impulse and aperture radar," in Proc. 2001 CIE International Conference on Radar, Oct. 2001, pp. 76-80.

[14]P. J. Gibson, "The Vivaldi aerial," in Proceedings of the 9th European Microwave Conference. Brighton, UK, 1979.

[15]"Authorization of Ultrawideband Technology, First Report and Order, Federal Communications Commission, February 14, 2002," Federal Communications Commission, Washington, DC, USA FCC 02-48, 14 February 2002.

[16]D. G. Brennan, "Linear diversity combining techniques,", Proc. IRE, vol. 47, pp. 1075-1101, 1959.

[17] G. J. Foschini and M. J. Gans, "On the limits of wireless communications in a fading environment," Wireless Pers Commun, vol. 6, pp. 311-335, 1998.

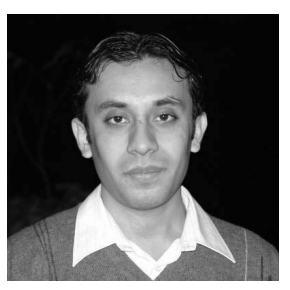

Hammad Khan was born on $15^{\text {th }}$ April 1980 in Gujranwala, Pakistan. He received his B.S. degree in Electronic Engineering from GIK Institute of Engineering Sciences and Technology, Topi, Pakistan, in 2002. He worked as a Software Engineer and a Project Management Consultant at Techlogix Pakistan Ltd. for a year before joining the Communications Research Group at the University of Oxford, UK where he is working towards a DPhil degree.

Mr. Khan is a member of IEEE, IEEE Communications Society and IEE. He has received a number of scholarships and awards including the Rhodes scholarship to study at Oxford. His research interests include radar theory and applications, electromagnetics and ultra wideband systems.

Wolfson College, Linton Road Oxford OX2 6UD, UK.

hammad.khan@eng.ox.ac.uk 